\title{
Antidiabetic drugs and the risk of cancer: beneficial, neutral, or detrimental?
}

\author{
Taoreed Adegoke Azeez ${ }^{1 *}$, Sharif Adeniyi Folorunso², \\ Chinedu Eguzozie ${ }^{1}$, Adeleke Adedapo Adegboyega ${ }^{3}$ \\ ${ }^{1}$ Endocrinology, Metabolism and Diabetes Unit, Department of Medicine, \\ University College Hospital, Ibadan, Nigeria \\ ${ }^{2}$ Department of Radiation Oncology, University College Hospital, \\ Ibadan, Nigeria \\ ${ }^{3}$ Department of Clinical Pharmacology, University College Hospital, \\ Ibadan, Nigeria
}

Received 4 August 2020; Accepted 21 September 2020

\begin{abstract}
The prevalence of diabetes mellitus is rapidly rising, especially in low- and middle-income countries. Also, early-onset diabetes is on the rise, and millions of individuals have to be on antidiabetic medications for a prolonged period. Therefore, more people are getting exposed to the adverse effects of antidiabetic medications.

Cancer is among the top ranking causes of death worldwide. Researches are still ongoing to understand the etiologies, precipitants, risk factors, correlates, and predictors of cancers. Diabetes mellitus is associated with various cancers, as extensively documented in the literature. There are conflicting reports about the association between antidiabetic drugs and cancer. This is even of crucial importance, considering that the prevalence of diabetes is rising.

Insulin glargine is reported to be associated with cancers, but clinical trials have not confirmed this. Metformin is largely believed to be beneficial in oncologic practice. Glibenclamide is reported to reduce tumor growth. The association between pioglitazone and bladder cancer is still an area for further research. Meglitinides have also been associated with cancers. Incretin-based therapy and the $\alpha$-glucosidase inhibitors appear to have beneficial effects on cancers.

There is still a need for randomized multicentric clinical trials to further substantiate and clarify reports from epidemiological studies. Further in vitro studies will also be necessary to characterize the interaction of these pharmacological agents with other molecules in the body.
\end{abstract}

Keywords: antidiabetic drugs • diabetes $•$ cancer

\section{Background}

Diabetes mellitus is a non-communicable disease in which there is a disorder of carbohydrate, lipid, and protein metabolism. ${ }^{[1]}$ It is defined as a chronic metabolic disorder characterized by hyperglycemia due to deficiency in insulin secretion and/or action. ${ }^{[2]}$ According to their 2019 classification of diabetes, which is the latest classification, diabetes is classified into type 1 diabetes, type 2 diabetes, hybrid diabetes, gestational diabetes, other specific types of diabetes, and the unclassified type. ${ }^{[3]}$ Going by the ninth edition of the diabetes atlas published by the
International Diabetes Federation (IDF), the global prevalence of diabetes is about 463 million, which corresponds to about $9.3 \%$ of the world's population. ${ }^{[4]}$ It is estimated that by 2045 , the prevalence would have risen to 700 million, which would be over $10 \%$ of the global population. ${ }^{[4]}$

Most individuals living with type 2 diabetes are on at least one antidiabetic medication. ${ }^{[5]}$ Interestingly, the onset of diabetes in most of these people is found in childhood to middle age, which translates that they still have a substantial amount of years to live with the disease and be on antidiabetic drugs ${ }^{[2]}$ Globally, several pharmacological classes of antidiabetic drugs are available to the diabetic 
population and many more are being discovered on a regular basis. Unfortunately, the carcinogenic potentials of these medications are still subjects of debate, with scanty and scattered literature to support or refute whichever hypothesis is being held. Although safety profiles are often required by drug regulatory bodies such as the Federal Drug Agency (FDA) in the USA and the European Medicines Agency (EMA), development of cancer requires a long period of follow-up. This may not be feasible in most cases and the ultimate reliance is on post-marketing surveys which have their own drawbacks. ${ }^{[5]}$

Cancers are a heterogeneous group of diseases with major public health implications. Cancer, generally, is the second leading cause of death worldwide, with the majority of deaths occurring in the low- and middleincome countries, the same areas where diabetes has the highest prevalence. ${ }^{[4],[6]}$ The literature is replete with reports of the association between diabetes and cancers including, liver, pancreas, breast, and colorectal cancers. ${ }^{[7]}$ People with diabetes are also more likely to die from cancer. Therefore, it is of crucial importance for clinicians to be aware of the relationship between antidiabetic drugs and cancers, so as not to unnecessarily increase the risk of developing cancers.

The various classes of antidiabetic drugs that are in common use include insulins, biguanides, sulfonylureas, thiazolidinediones, a-glucosidase inhibitors, meglitinides, and amylin mimetics. Others are dipeptidyl peptidase-4 (DPP-4) inhibitors, glucagonlike peptide-1 (GLP-1) agonists, and sodium-glucose co-transporters-2 (SGLT-2) inhibitors. ${ }^{[8]}$ Some of the drugs in each class have been implicated in cancers, some have been stated to be beneficial, some have no beneficial or detrimental effect on carcinogenesis, while the literature is very scanty on some as far as their association with cancers is concerned. The aim of this review is to highlight how the antidiabetic medications are related to cancers.

\section{Insulin therapy and cancer}

It is estimated that about $20 \%-30 \%$ of the patients with diabetes are on one form of insulin therapy or the other. [9] Insulin is the oldest form of treatment for diabetes. Insulin therapy is absolutely indicated in type 1 diabetes, while many patients with type 2 diabetes are also on insulin. The common insulin preparations in usage include regular insulin, lispro, aspart, glulisine, neutral protamine Hagedorn (NPH) insulin, glargine, detemir, and degludec. ${ }^{[10]}$ Aspart, lispro, and glulisine are called rapidly acting insulin, regular insulin is called short-acting insulin, NPH insulin is an intermediate-acting insulin, glargine and detemir are long acting, while degludec is ultra-long acting. Modes of administering insulin include the use of syringe, pen, prefilled pen, and pump. Inhaled insulins have been developed, but they are rarely used. Soluble insulin and NPH insulin are referred to as human insulins, whereas others are analogs.

Insulin can act as a growth factor via the mitogenic pathway, causing proliferation of different types of cells. ${ }^{[11]}$ The effect of insulin is mitogenic (stimulating cell growth) and not mutagenic (inducing cell transformation), unlike insulin-like growth factor-1 (IGF-1). ${ }^{[12]}$ Many cancer cells express IGF-1 receptors which have low affinity for insulin; but in the presence of hyperinsulinemia in insulin resistance, the effect of insulin on the receptor could become significant. ${ }^{[13]}$

Studies on the association between the use of insulin and the risk of developing cancer have reported mixed findings. ${ }^{[12]}$ Epidemiological studies have suggested an increased risk of cancer in patients using insulin. ${ }^{[14]}$ It is worthy of note that some studies did not report any increased risk of cancer with insulin therapy. ${ }^{[15]}$ In fact, a study reported a protective effect of insulin therapy on the risk of developing cancer. ${ }^{[16]}$ This heterogeneity does not necessarily mean that there are technical flaws with the mixed findings, but may suggest that different cancers behave in diverse ways with exposure to insulin. [12] There could also be multiple cofounders, which are a major drawback of epidemiological studies.

Direct and extrapolated data from randomized control trials, such as the Outcome Reduction with an Initial Glargine Intervention (ORIGIN) trial, the Bypass Angioplasty Revascularization Investigation 2 Diabetes (BARI 2D), and the Action to Control Cardiovascular Risk in Diabetes (ACCORD) trial, show that there is no evidence of increased risk of cancer with insulin therapy compared with other common medications. ${ }^{[12],[17]}$ However, the doses of insulin used in these studies may not be adequate and of sufficient duration because in vitro and epidemiological studies have suggested that effect of insulin on cell proliferation is dose and duration dependent. ${ }^{[13]}$

Comparing human insulins with the analogs, it has been found that the analogs have higher affinity and a lower dissociation rate from the IGF-1 receptors than the human insulins. ${ }^{[12]}$ However, there is no evidence that the analogs have higher mitogenic potential than the human insulins, with a notable exception of glargine. ${ }^{[18]}$ Glargine has demonstrated an increased mitogenic potential with some cancers such as prostate and colorectal cancers, but this was not observed in other cancers such as pancreatic and thyroid cancers. ${ }^{[12]}$ However, a study has suggested that the mitogenic potential of glargine is expressed only at higher doses and with prolonged 
duration. ${ }^{[19]}$ It must, however, be stated that most of these findings on glargine were from in vitro and epidemiologic studies which have multiple drawbacks. A randomized controlled trial, ORIGIN trial, did not demonstrate increased risk of any cancer with the use of glargine.

\section{Biguanides and cancer}

Biguanides are a class of oral glucose-lowering agents derived from guanidine. Members of this class are metformin (dimethylbiguanide), phenformin (phenethylbiguanide), and buformin (butylbiguanide). Phenformin and buformin have been withdrawn from the markets in the late 1970s due to their toxic effects, especially lactic acidosis. ${ }^{[20]}$ Biguanides do not cause insulin release and rarely cause hypoglycemia. So, they are referred to as anti-hyperglycemic drugs rather than hypoglycemic agents.

The most commonly prescribed oral glucoselowering agent is metformin. ${ }^{[21]}$ Its mechanism of action is to decrease hepatic glucose output and increase insulin sensitivity in the skeletal muscles. ${ }^{[2]}$ Metformin impairs adenosine triphosphate (ATP) production in the mitochondria and this leads to the activation of the adenosine monophosphate-activated protein kinase (AMPK) pathway. ${ }^{[23]}$ This pathway is important in the overall regulation of energy homeostasis at the cellular level. The activation of this pathway leads to downregulation of various cellular processes that consume ATP, such as protein synthesis and fatty acid biosynthesis, eventually restoring ATP to its previous levels.

The earliest indication to suggest that metformin may play a role in oncology was from various epidemiological studies which found lower mortality among patients with diabetes and cancer taking metformin compared with other glucose-lowering agents. ${ }^{[24]}$ It is known that hyperinsulinemia can cause proliferation of cells, including cancer cells (in vitro studies); hence, it is hypothesized that the antineoplastic effect of metformin may be linked to its ability to reduce hyperinsulinemia. [25] It has also been found that that it can sensitize the cancer cells to the antineoplastic effect of the chemotherapy agents. ${ }^{[26]}$ The main drawbacks with this study were that the level of evidence was very low as it was a retrospective observational study and the sample size was also very small.

Chronic low-grade systemic inflammation has been found to enhance carcinogenesis, cancer growth, and metastasis. ${ }^{[27]}$ Metformin has been found to reduce the production of inflammatory mediators, and it is hypothesized that this may be the explanation for the antineoplastic effect of metformin. ${ }^{[28]}$ Radiotherapy and chemotherapeutic agents such as cisplatin act by causing deoxyribonucleic acid (DNA) damage, and some researchers have suggested that metformin can enhance this DNA damaging effect. [29]

Some clinical trials have also shown some beneficial effects of metformin in cancer patients. Using Ki-67 staining as a marker of tumor cell proliferation, it was demonstrated that metformin induced a small decline in tumor cell proliferation when administered to nondiabetic women with operable breast cancer. ${ }^{[30]} \mathrm{A}$ similar finding has been reported on colon cancer. ${ }^{[31]}$

While most of the documented literature on the association between usage of metformin and cancer points toward the antineoplastic effect of metformin, a few studies have reported the increased risk of tumor growth with metformin administration. The main mechanism of action is by activating the AMPK pathway. ${ }^{\text {[23] }}$ Activation of this pathway has, however, been found to lead to increased expression of the vascular endothelial growth factor (VEGF) which is linked to tumor growth. [32] However, a study comparing co-administration of an anti-VEGF agent with metformin with each agent independently found a better antineoplastic effect with the combined medication. ${ }^{[32]}$

\section{Sulfonylureas and cancer}

Sulfonylureas, as a group of antidiabetic agents, act by stimulating insulin secretion in the pancreatic $\beta$ cells. ${ }^{[33]}$ First-generation sulfonylureas include chlorpropamide, acetohexamide, and tolbutamide. Second-generation sulfonylureas include glibenclamide, glipizide, and gliclazide. Glimepiride is sometimes classified as a thirdgeneration sulfonylurea, while some authors still classify it as a second-generation sulfonylurea. Sulfonylureas act on the sulfonylurea receptor (SUR), which is a subunit of the ATP-sensitive potassium channels $\left(\mathrm{K}_{\text {ATP }}\right)$, leading to the secretion of insulin from the $\beta$ cells. ${ }^{[34]}$

Epidemiological studies have shown some evidence that glibenclamide can reduce tumor growth. ${ }^{[35]}$ Some cancers such as gastric cancer have been shown to express $\mathrm{K}_{\text {ATP }}$, and the ability of glibenclamide to close these potassium channels has been implicated as the mechanism behind the tumor antiproliferative effect of glibenclamide. ${ }^{[36]}$ Glibenclamide has also been reported to be associated with increased generation of reactive oxygen species by cancer cells, and this may induce apoptosis of the cancer cells. ${ }^{[37]}$

Most of the other sulfonylureas have not demonstrated any effects on cancer. ${ }^{[38]}$ However, there are a few reports on gliclazide. Gliclazide is different from other sulfonylureas because it binds to SUR in 
a rapidly reversible manner. It reduces the activity of reactive oxygen species and aids in genomic stability and DNA repair. ${ }^{[39]}$ A study on pancreatic cancer found a positive effect on the ability of gliclazide to aid in cancer cells' DNA repair. ${ }^{[40]}$

\section{Thiazolidinediones and cancer}

Thiazolidinediones (also called glitazones) are a group of glucose-lowering agents which act on a nuclear receptor called peroxisome-proliferator activated receptor gamma (PPARY). ${ }^{[41]}$ The most commonly used glitazones are pioglitazone and rosiglitazone. Others include ciglatozone and troglitazone. They improve insulin sensitivity in adipose tissue and muscle. They also reduce hepatic glucose output. Some of the side effects of glitazones are weight gain, edema, hepatotoxicity, and osteoporosis.

Epidemiological studies have shown some beneficial antineoplastic effects of glitazones in lung, breast, and colon cancers. ${ }^{[42]}$ There are reports that they can upregulate the expression of tumor suppression genes and downregulate the genes involved in cell proliferation and cell cycle. ${ }^{[43]}$ Induction of apoptosis in tumor cell has also been documented as an antineoplastic mechanism of the glitazones. ${ }^{[44]}$ They also promote differentiation of cells into the normal phenotype. Glitazones have also been reported to reduce VEGF levels in hyperinsulinemic rats. ${ }^{[45]}$ VEGF is involved in angiogenesis, and its overexpression plays an important role in the metastasis of cancer. A meta-analysis on the association between the risk of cancer and glitazones showed that pioglitazone and rosiglitazone were associated with reduced risk of cancers. ${ }^{[46]}$

In contrast, a longitudinal cohort study in Northern California, with a sample size of close to 200,000 individuals, reported an association between pioglitazone and bladder cancer. ${ }^{[47]}$ Another case-control study that recruited a total of about 20,000 individuals found an association between bladder cancer and the use of pioglitazone and rosiglitazone. ${ }^{[48]}$ Two separate meta-analyses also showed slightly increased risk of bladder cancer with thiazolidinediones, especially with pioglitazone. ${ }^{[49]}$

\section{Meglitinides and cancer}

Meglitinides are insulin secretagogues with a shorter duration of action compared with sulfonylureas. Examples include repaglinide, mitiglinide, and nateglinide. They are oral agents administered preprandially and tend to mimic the insulin secretion physiology better than sulfonylureas. Hence, they have a lesser risk of hypoglycemia. ${ }^{[50]}$ This is possible because they bind weakly with SUR and they can dissociate much rapidly. ${ }^{[51]}$

There is scanty literature on the link between meglitinides and cancers. A retrospective study observed increased cancer risk, especially hepatocellular cancer, among patients on meglitinides. ${ }^{[52]}$ According to the authors, the proposed explanation relates with hyperinsulinemia caused by the meglitinides, and hyperinsulinemia has been repeatedly associated with cancers. A hospital-based case-control study examined the association between usage of glucose-lowering agents among diabetic patients and the risk of pancreatic cancer and found that meglitinide administration was associated with an increased risk of pancreatic cancer. ${ }^{[53]}$

\section{DPP-4 inhibitors and cancers}

DPP-4 inhibitors are a group of oral glucose-lowering agents that inhibit an enzyme called dipeptidyl dipeptidase. The enzyme breaks down some molecules called incretins, examples of which include GLP-1 and glucose-dependent insulinotropic polypeptide (GIP). [54] These incretins have beneficial effects such as decreasing glucagon release which leads to increased insulin secretion, suppressing appetite, and delaying gastric emptying. All these mechanisms ultimately lead to decreased blood glucose. Examples include sitagliptin, saxagliptin, alogliptin, vildagliptin, and linagliptin.

A meta-analysis has reported non-statistically significant association between DPP-4 inhibitors and breast, thyroid, and pancreatic cancers. ${ }^{[55]}$ DPP-4 is ubiquitous and it is found in many tissues and body fluids. It can act as a tumor suppressor or activator depending on the chemical milieu. ${ }^{[56]}$ In contrast, another study demonstrated a survival advantage for patients with diabetes and colorectal cancer or lung cancer who were on DPP-4 inhibitors. ${ }^{[57]}$ When $T$ lymphocytes invade tumor, in order to destroy it as a form of immune reaction against the tumor, they secrete chemokines. ${ }^{[57]}$ The enzyme dipeptidyl peptidase degrades the chemokines. It is thought that when DPP-4 inhibits the degradation of the chemokines, it can enhance an optimal immune response against the tumor cells.

\section{GLP-1 agonists and cancers}

Examples of drugs in this group are exenatide, dulaglutide, liraglutide, semaglutide, and lixisenatide. 
GLP-1 is an incretin whose physiological roles include reduction of glucagon secretion, enhanced satiety, and delayed gastric emptying. GLP-1 agonists mimic these physiological functions and eventually lower blood glucose. ${ }^{[58]}$

There are reports linking GLP-1 receptor agonists with malignant neoplasia, especially pancreatic cancer and thyroid c-cell cancer. ${ }^{[59]}$ It is reported that chronic stimulation of the GLP-1 receptor causes inflammation of the organ, pancreatitis for example, which increases the risk of developing cancer. ${ }^{[60]}$ However, a metaanalysis did not find any increased incidence of cancer in patients on GLP-1 receptor agonists compared with placebo or other agents. ${ }^{[61]}$

\section{SGLT-2 inhibitors and cancers}

SGLT-2 inhibitors are a class of glucose-lowering agents whose mechanism of action is to impair reabsorption of glucose from the renal tubules, thereby enhancing glucose excretion in the urine, hence lowering the plasma glucose. Examples are dapaglifozin, canaglifozin, and empaglifozin.

A meta-analysis reported that there is no increased incidence of cancers associated with SGLT-2 inhibitors. ${ }^{\left[{ }^{62]}\right.}$ A study done in rodents also showed that dapaglifozin slows tumor growth in breast and colon cancers. ${ }^{[63]}$ It was documented that the ability of dapaglifozin to cause weight loss would lower hyperinsulinemia, which has been richly documented to be associated with malignant neoplasia. ${ }^{[63]}$ Ipraglifozin was also reported to have induced apoptosis of breast cancer cells. ${ }^{[64]}$

\section{References}

[1] Asiimwe D, Mauti GO, Kiconco R. Prevalence and risk factors associated with type 2 diabetes in elderly patients aged 45-80 years in Kanungu district. J Diabetes Res. 2020; 2020: 5152146.

[2] American Diabetes Association (ADA), "Classification and diagnosis of diabetes: standards of medical care in diabetes," Diabetes Care. 2019; 42, (5): 13-28.

[3] World Health Organization (WHO). Classification of diabetes mellitus. WHO. 2019; pp8.

[4] Saeedi P, Petersohn I, Salpea P, Malanda B, Karuranga S, Unwin $N$ et al. Global and regional diabetes prevalence estimates for 2019 and projections for 2030 and 2045. Diabetes Res Clin Pract. 2019. 2019: 107843.

\section{0. $\alpha$-Glucosidase inhibitors and cancers}

a-Glucosidase inhibitors delay the absorption of carbohydrates in the small intestine. They target postprandial glucose excursion. Examples are acarbose, miglitol, and voglibose. A meta-analysis showed that a-glucosidase inhibitors are associated with reduced risk of having cancers. ${ }^{[6]}$ The mechanistic explanation for this observation was the ability of a-glucosidase inhibitors to lower postprandial hyperinsulinemia, which has been linked with cancers. ${ }^{[66]}$

\section{Conclusion}

Diabetes mellitus is a chronic metabolic disorder which has been reportedly associated with cancers in several studies. Therefore, there is a need to highlight the antidiabetic medications that are associated with cancers. The literature has conflicting information on the association of various antidiabetic medications and cancers. This neoplastic tendency is also not uniform across the group. While some glucose-lowering medications have been extensively documented to be beneficial, some are neutral, and there are substantial reports that call for caution on some of them. There is a need for randomized clinical trials to further expatiate on these relationships between antidiabetic medications and cancers.

[5] Liu, YC., Nguyen, PA., Humayun, A., Chien, SC. Yang, HC.et al.). Does long-term use of antidiabetic drugs changes cancer risk? Medicine, 2019; 98(40), e17461.

[6] Siegel RL, Miller KD, Jemal A. Cancer statistics, 2019. Cancer. 2019; 69(1): 7-34.

[7] Abudawood M. Diabetes and cacer-a comprehensive review. J Res Med Sci. 2019; 24:94.

[8] Bailey CJ. The Current Drug Treatment Landscape for Diabetes and Perspectives for the Future. Clin Pharmacol Ther. 2015;98(2):170-184.

[9] Turner LW, Nartey D, Stafford RS, Singh S, Alexander GC. Ambulatory treatment of type 2 diabetes in the U.S., 1997-2012.Diabetes Care. 2014; 37(4):985-92 
[10] Steven H, Barag DO. Insulin therapy for management of type 2 diabetes mellitus: strategies for initiation and long term patient adherence. J Am Osteopath Assoc. 2011; 111: S3-S11.

[11] Pollak M. The insulin and insulin-like growth factor receptor family in neoplasia: an update. Nat Rev Cancer. 2012; 12(3): 159-169.

[12] Mannucci E. Insulin therapy and cancer in type 2 diabetes. Int Sch Ret Notices. 2012; 2012: 240634.

[13] Vigneri P, Frasca F, Sciacca L, Pandini G, Vigneri R. Diabetes and cancer. Endocrine-Related Cancer. 2009; 16(4): 1103-1123.

[14] Yang YX, Hennessy S, Lewis JD. Insulin therapy and colorectal cancer risk among type 2 diabetes mellitus patients. Gastroenterology 2004; 127(4): 1044-1050.

[15] Jonasson JM, Ljung R, Talbäck M, Haglund N, Gudbjörnsdòttir S, Steineck J. Insulin glargine use and short-term incidence of malignancies-a population-based follow-up study in Sweden. Diabetologia,. 2009; 52(9): 1745-1754.

[16] Yang X, Ko GTC, So W. Associations of hyperglycemia and insulin usage with the risk of cancer in type 2 diabetes: the Hong Kong diabetes registry. Diabetes. 2010; 59(5): 1254-1260.

[17] ORIGIN Trial Investigators, Gerstein HC, Bosch J. Basal insulin and cardiovascular and other outcomes in dysglycemia. The New England Journal of Medicine. 2012; 367(4): 319-328.

[18] Staiger K, Hennige AM, Staiger $H$, Häring $H U$, Kellerer M. Comparison of the mitogenic potency of regular human insulin and its analogue glargine in normal and transformed human breast epithelial cells. Hormone and Metabolic Research. 2007; 39(1): 65-67.

[19] Morden NE, Liu SK, Smith J, Mackenzie TM, Skinner J, Korc M. Further exploration of the relationship between insulin glargine and incident cancer: a retrospective cohort study of older Medicare patients. Diabetes Care. 2011; 34(9): 1965-1971

[20] Wang GS, Hoyte C. Review of biguanides (metformin) toxicity. J Intensive Care Med. 2018; 34(11): 1177.

[21] American Diabetes Association. Prevention or delay of type 2 diabetes: standards of medical care in diabetes. Diabetes Care. 2019; 42 (Suppl.1): 529-533.

[22] Hotta N. A new perspective on the biguanides, metformin therapy in type 2 diabetes and lactic acidosis. J Diabetes Investig. 2019; 10(4): 906-908.

[23] Hawley SA, Ross FA, Chevtzoff C. Use of cells expressing gamma subunit variants to identify diverse mechanisms of AMPK activation. Cell Metab. 2010; 11:554-65.

[24] Pollak M. Metformin and other biguanides in oncology: advancing the research agenda. Cancer Prev Res. 2010; 3(9): 1158.

[25] Algire C, Zakikhani M, Blouin M-J, Shuai JH, Pollak M. Metformin attenuates the stimulatory effect of a high energy diet on in vivo H59 carcinoma growth. Endocr Relat Cancer. 2008; 15:833-839.

[26] Bodmer M, Meier C, Krahenbuhl S, Jick SS, Meier CR, Meier CR. Long term metformin use is associated with decreased risk of breast cancer. Diabetes Care. 2010; 33:1304-8.

[27] Multhoff G, Molls M, Radons J. Chronic inflammation in cancer development. Front Immunol. 2011; 2: 98.

[28] Polak M. Potential application of biguanides in oncology. J Clin Invest. 2013; 2013: 167232.

[29] Zong WX, Ditsworth D, Bauer DE, Wang ZQ, Thompson CB. Alkylating DNA damage stimulates a regulated form of necrotic cell death. Genes Dev. 2004;18(11):1272-1282.

[30] Bonanni B. Dual effect of metformin on breast cancer proliferation in a randomized presurgical trial. J Clin Oncol. 2012;30(21):2593-2600.

[31] Hosono K. Metformin suppresses colorectal aberrant crypt foci in a short-term clinical trial. Cancer Prev Res. 2010;3(9):1077-1083.

[32] Martin MJ, Hayward R, Viros A, Marais R. Metformin accelerates the growth of BRAF V600Edriven melanoma by upregulating VEGF-A. Cancer Discov. 2012;2(4):344-355.

[33] Seino S. Cell signalling in insulin secretion: the molecular targets of ATP, cAMP and sulfonylurea. Diabetologia. 2012; 55 (8): 2096-2108

[34] Proks P, Reimann F, Green N, Gribble F, Ashcroft F. Sulfonylurea stimulation of insulin secretion. Diabetes. 2002; 51 (Suppl 3): S368-376.

[35] Núñez M, Medina V, Cricco G, Croci M, Cocca C, Rivera $\mathrm{E}$ et al. Glibenclamide inhibits cell growth by inducing G0/G1 arrest in the human breast cancer cell line MDA-MB-231. BMC Pharmacol Toxicol. 2013; 14(1):6

[36] Ardehali H, O'Rourke B. Mitochondrial K(ATP) channels in cell survival and death. $J \mathrm{Mol}$ Cell Cardiol. 2005; 39(1):7-16

[37] Qian X, Li J, Ding J, Wang Z, Duan L, Hu G. Glibenclamide exerts an antitumor activity through reactive oxygen species-c-jun $\mathrm{NH} 2$-terminal kinase pathway in human gastric cancer cell line MGC803. Biochem Pharmacol. 2008; 76(12):1705-15

[38] Pasello G, Urso L, Conte P, Favarreto. Effects of sulphonylureas on tumor growth: a eview of the literature. Oncologist. 2013; 18(10):118-1125. 
[39] Pan HZ, Chang D, Feng LG, Xu FJ, Kuang HY, Lu MJ. Oxidative damage to DNA and its relationship with diabetic complications. Biomed Environ Sci. 2007; 20(2):160-3.

[40] Sliwinska A, Sliwinski T, Kasznicki J, Drzewoski J. Effect of gliclazide on nucleotide excision repair (NER) and non-homologous DNA end joining (NHEJ) in normal and cancer cells. J Physiol Pharmacol. 2010; 61(3):347-53.

[41] Ferruzzi P, Ceni E, Tarocchi M, Grappone C, Milani S, Galli A et al. Thiazolidiediones inhibit the growth and invasiveness of adrenocortical cancer cell line H295R. J Clin Endocrinol Metab. 2005; 19(3): 1332-1339.

[42] Blanquicett C, Roman J, HartCM. Thiazolidinediones as anti-cancer agents. Cancer Ther. 2008; 6(A): 2534.

[43] Vignati S, Albertini V, Rinaldi A, Kwee I, Riva $C$, Oldrini $R$ et al. Cellular and molecular consequences of peroxisome proliferator-activated receptor-gamma activation in ovarian cancer cells. Neoplasia. 2006; 8(10):851-61.

[44] Clay CE, Namen AM, Atsumi G, Willingham MC, High KP, Kute TE et al. Influence of $\mathrm{J}$ series prostaglandins on apoptosis and tumorigenesis of breast cancer cells. Carcinogenesis. 1999; 20(10):1905-11

[45] Yang B, Lin P, Carrick KM, McNulty JA, Clifton LG, Winegar DA et al. PPARgamma agonists diminish serum VEGF elevation in diet-induced insulin resistant SD rats and ZDF rats. Biochem Biophys Res Commun. 2005; 334(1):176-82

[46] Monami M, Dicembrini I, Mannucci E. Thiazolidinediones and cancers: reults of a metaanalysis of randomized clinical trials. Acta Diabetol. 2014; 51(1): 91-101.

[47] Lewis J. D., Ferrara A., Peng T. et al. Risk of bladder cancer among diabetic patients treated with pioglitazone: interim report of a longitudinal cohort study. Diabetes Care. 2011; 34 (4): 916-922.

[48] Hsiao F.-Y., Hsieh P.-H., Huang W.-F., Tsai Y.W., Gau C.-S. Risk of bladder cancer in diabetic patients treated with rosiglitazone or pioglitazone: A Nested Case-control Study. Drug Safety. 2013; 36(8):b643-649.

[49] Chiu M, McBeth L, Sindhwani P, Hinds TD. Deciphering the roles of thiazolidinediones and PPARy in bladder cancer. PPAR Res. 2017; 2017: 4810672.

[50] Bellomo DA, Stefanelli G, Laviola L, Giorgino R, Giorgino F. Nateglinide provides tither glycemic control than glyburide in patients with type 2 diabetes with prevalent postprandial hyperglycemia. Diabet Med, 2011; 28(5): 560-65.
[51] Blicklé JF. Meglitinide analogues: a review of clinical data focused on recent trials". Diabetes Metab. 2006; 32 (2): 113-20

[52] Chang $\mathrm{CH}$, Lin JW, Wu LC, Lai MS, Chuang LM. Oral insulin secretagogues, insulin, and cancer risk in type 2 diabetes mellitus. J Clin Endocrinol Metab. 2012; 97(7):E1170-5.

[53] Li D, Yeung SJ, Hassan MM, Konopleva M, Abruzesse JL. Anti-diabetic therapies affect the risk of pancreatic cancer. Gastroenetrology. 2009; 137(2): 482-488.

[54] Mclntosh, C; Demuth, H; Pospisilik, J; Pederson, R (). Dipeptidyl peptidase IV inhibitors: How do they work as new antidiabetic agents?. Regulatory Peptides. 2005; 128 (2): 159-65.

[55] Overbeek JA, Bakker M, van der Heijden AAW, van Herk-Sukel MPP, Herings RMC, Nijpels G. Risk of dipeptidyl peptidase-4 (DPP-4) inhibitors on site-specific cancer: A systematic review and meta-analysis. Diabetes Metab Res Rev. 2018; 34(5): $\mathrm{e} 3004$

[56] Urasaki Y, Ohnuma K, Morimoto C, Dang N. The role of CD26/dipeptidyl peptidase IV in cancer. Front Biosci. 2008;13:1634-1645.

[57] Bishnoi R, Hong Y, Shah C, Azka A, Skelton WP, Huo $J$. et al. Dipeptidyl peptidase 4 inhibitors as novel agents in improving survival in diabetic patients with colorectal cancer and lung cancer: A Surveillance Epidemiology and Endpoint Research Medicare study. Cancer Med. 2019; 8(8): 3918-3927.

[58] Hinnen D. Glucagon-like 1 peptides receptor for type 2 diabetes. Diabetes spectrum. 2017; 30(3): 202-210.

[59] Bjerre Knudsen L, Madsen LW, Andersen S. Glucagon-like peptide-1 receptor agonists activate rodent thyroid $\mathrm{C}$-cells causing calcitonin release and C-cell proliferation. Endocrinology. 2010; 151 (4): 1473-1486.

[60] Chalmer T, Almdal TP, Vilsbøll T, Knop FP. Adverse drug reactions associated with the use of liraglutide in patients with type 2 diabetes - focus on pancreatitis and pancreas cancer. Expert Opinion on Drug Safety. 2015; 14(1): 171-180.

[61] Liu Y, Zhang X, Chao S, Zhao X, Ji L. Risk of Malignant Neoplasia with Glucagon-Like Peptide-1 Receptor Agonist Treatment in Patients with Type 2 Diabetes: A Meta-Analysis. J Diabetes Res. 2019; 2019: 1534365.

[62] Dicembrini I, Nreu B, Mannucci E, Monami M. Sodium-Glucose Co-Transporter-2 (SGLT2) Inhibitors and Cancer: A Meta-Analysis of Randomized Controlled Trials. Diabetes Obes Metab 2019; 21(8)1871-1877. 
[63] Nasiri Al, Rodrigues MI, Li Z, Leitner BP, Perry RJ. SGLT2 inhibition slows tumor growth in mice by reversing hyperinsulinemia. Cancer Metab. 2019; 7: 10.

[64] Komatsu S, Nomiyama T, Numata T, Kawanami T, Hamaguchi $Y$, Tanaka T et al. SGLT2 Inhibitor Ipragliflozin Induces Breast Cancer Apoptosis via Membrane Hyperpolarization and Mitochondria Dysfunction. Diabetes. 2018; 67 (Suppl 1): 255

[65] Zhao Y, Wang Y, Lou H, Shan L. Alpha-glucosidase inhibitors and risk of cancer in patients with diabetes mellitus: a systematic review and meta-analysis. Oncotarget. 2017; 8(46): 81027-81039.

[66] Inoue I, Takahashi K, Noji S, Awata T, Negishi K, Katayama S. Acarbose controls postprandial hyperproinsulinemia in non-insulin dependent diabetes mellitus. Diabetes Res Clin Pract. 1997; 36(3):143-5 